\title{
THE POSSIBLE ROLE OF SURGERY IN THE TREATMENT OF ANOMALOUS LEFT CORONARY ARTERY
}

BY

\author{
J. APLEY, R. E. HORTON, AND M. G. WILSON \\ From the Departments of Surgery and Child Health, University of Bristol
}

(RECEIVED FOR PUBliCATION JULY 9, 1956)

The rare anomaly in which the left coronary artery arises from the pulmonary trunk instead of from the aorta is the basis of a natural experiment of great interest. It provides the conditions for studying a local and gradually developing ischaemic reaction in the area of heart muscle served by the anomalous artery, and a general and compensatory reaction of the remainder of the heart. As a pathological curiosity it was first described in 1911 by Abrikossoff, but not until 1933 was a clear clinical picture of the condition given by Bland, White, and Garland. In a recent review Kittle, Diehl, and Heilbrunn (1955) collected 42 cases from the literature. In the early cases diagnosis was made at necropsy, but in more than half the cases reported recently the correct diagnosis has been reached on clinical findings. In this country we have been able to find reports of only three cases, two of which were not diagnosed until after death (Barnard, 1938; Fisher, and Lloyd, 1951). In addition Brown's presumed case (1950) has survived to adult life and necropsy confirmation is not available. Now that the clinical picture is becoming established ante-mortem diagnosis should be the rule, and, since very few cases are believed to survive the first year of life, the possibility of treatment by surgery must be seriously considered.

The characteristic history is that of an infant developing symptoms in the first weeks or months of life. Typically, respirations are rapid, and there is difficulty in feeding, with failure to thrive. Vomiting is frequent. Superimposed on this nonspecific picture there are in most cases episodes resembling anginal attacks in adults. The frequency of these attacks tends to increase progressively until death. An attack is usually provoked by feeding. The infant suddenly appears apprehensive, and lies still with the legs pulled up on the chest. Breathing seems almost imperceptible. He is pale, possibly with a cyanotic tinge. After a few minutes he screams as if in pain, then lies limp, exhausted, and covered in perspiration.
On clinical examination gross enlargement of the heart is found. Murmurs are unusual and unhelpful, and signs of cardiac failure are rarely detected. Radiology confirms the cardiac enlargement. The left ventricle is mainly affected; its pulsations are reduced and an aneurysm may be observed. The electrocardiogram resembles that of anterior infarction in adults. In addition to tachycardia the characteristic findings are: in leads I and V5 a deep $Q$ wave and S-T elevation with $\mathrm{T}$-wave inversion; reciprocal changes in lead III, with S-T depression and upright $\mathrm{T}$-waves. These features, particularly the electrocardiographic changes, nearly always permit differentiation from other causes of infantile cardiac hypertrophy.

In the case reported here, operation was attempted. It proved unsuccessful, but from the knowledge gained a procedure is suggested which may lead to successful attempts in the future.

\section{CASE REPORT}

A baby girl was admitted to the Bristol Children's Hospital at the age of 11 months. She was the third child of a healthy family. Gestation had been uneventful, birth was natural, and the birth weight was $9 \mathrm{lb}$. From birth she had been observed to breathe rapidly. She was breast-fed until 3 months, but always had difficulty in feeding. At 7 months and again at 8 months she was admitted to another hospital because of poor gain in weight and occasional vomiting, more marked since the age of 5 months, and improved on both occasions in hospital. It was observed that breathing was much more rapid after feeds.

On examination at 11 months she was underweight $(15 \mathrm{lb} .8 \mathrm{oz}$.) $(7 \mathrm{~kg}$.). There was no cyanosis. Marked chest recession occurred on inspiration, and the heart was clinically enlarged. A surprising feature was that a finger placed under the xiphisternum could feel distinctly the cardiac impulse coming from under the child's left costal margin, a sign which is unique in our experience of infants. There was a moderate but variable tachycardia. The first sound at the apex was 
loud and booming, and the second apical sound short and slightly accentuated. The pulmonary second sound was of average intensity and slightly split. Occasionally a faint, poorly localized systolic murmur was heard. The liver edge was palpable, but not tender. The child was seen to be cyanosed only once, when venepuncture was being done.

Cardioscopy demonstrated gross enlargement, especially of the left ventricle (Fig. 1). The pulsations of the left ventricle were barely discernible, and the possibility of an aneurysm was considered.

Electrocardiograms (Fig. 2) showed the characteristic changes described above.

During five weeks' stay in hospital the infant's condition fluctuated. At times she was restless, irritable, and difficult to feed; at other times she seemed quiet and contented. Weight gain was poor. Apart from an increase in the pulse and respiration rates no untoward results of feeding were observed. On one occasion she developed what appeared to be a respiratory infection, with fever, an abrupt rise in the respiration rate, and diminished air entry with harsh breath sounds at the left lung base. For a short time a gallop rhythm was heard in the heart. She re-

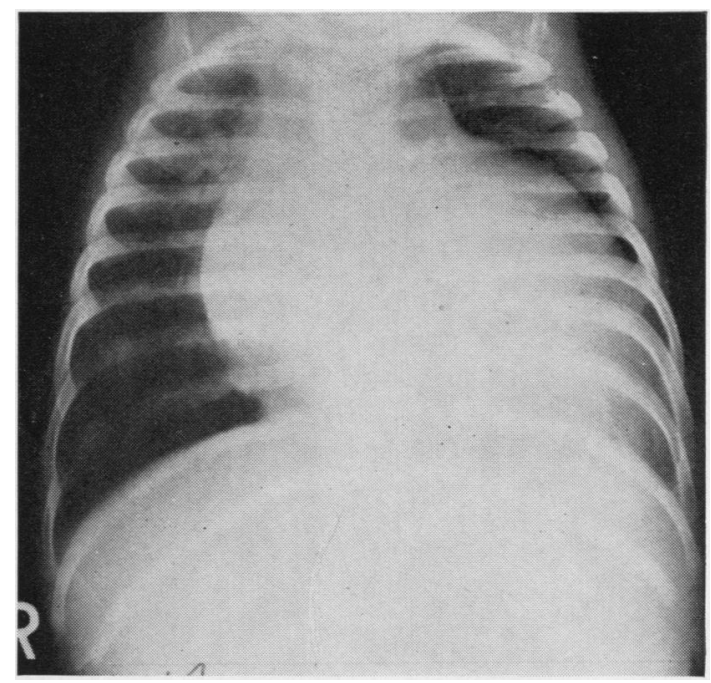

FIG. 1.-Radiograph of the chest, showing gross left ventricular enlargement.

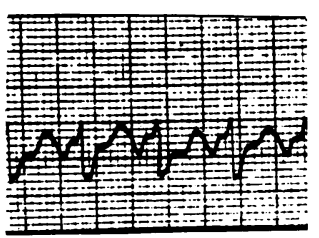

$\mathrm{aVr}$

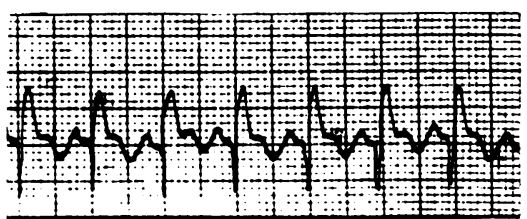

LEAD I

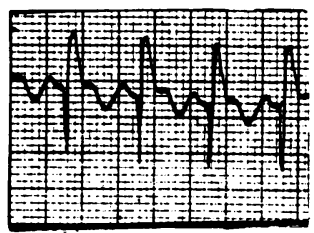

aVI

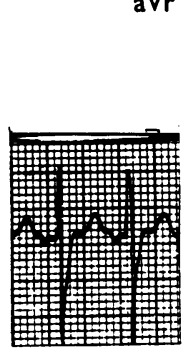

VI

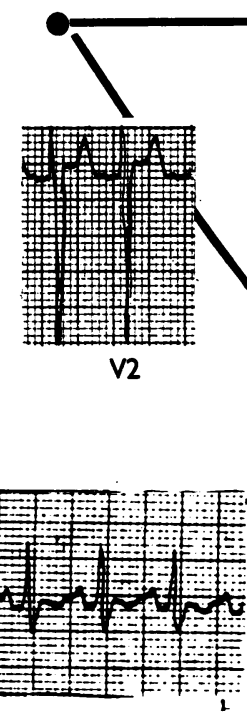

LEAD 2

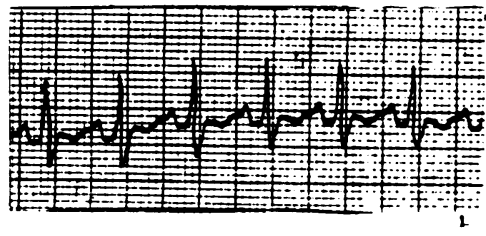

Fig. 2.-Electrocardiogram, in which leads 1 and V5 show deep Q waves, $\mathrm{S}-\mathrm{T}$ elevation and inversion of the $\mathrm{T}$ wave. Lead 3 shows reciprccal changes. 


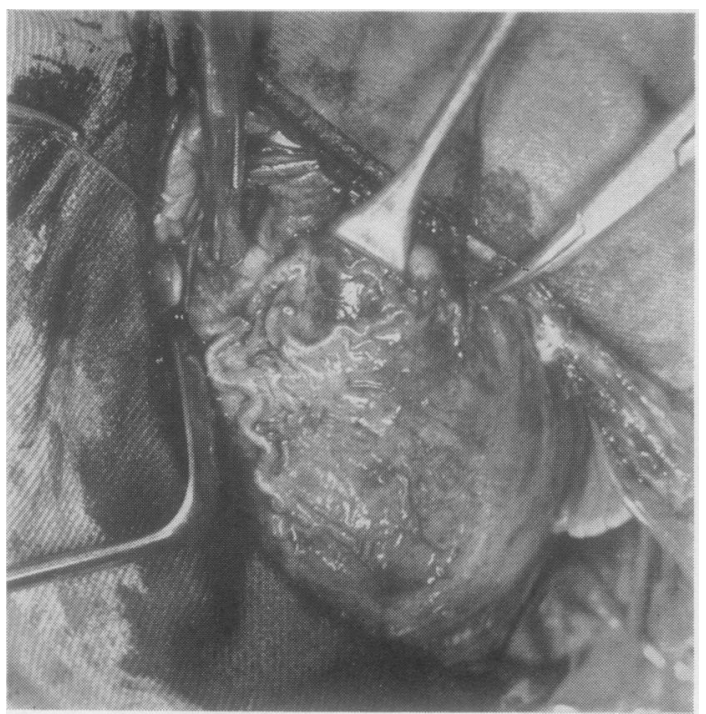

FIG. 3.-Photograph taken at operation showing large size and marked tortuosity of the right coronary artery.

sponded in a few days to aureomycin, digitalis, and tube-feeding.

At 1 year her general condition was as good as it had ever been while under medical attention, but her prospects of survival for more than a few months were considered to be negligible. It was therefore decided to try to correct the abnormality by an operation, the object of which was to detach the left coronary artery from the pulmonary artery and to anastomose to it the left subclavian artery. It was considered that the coronary artery would require perfusion during the anastomosis, and arrangements were made to tap the aorta with a polythene tube for this purpose.

OPERATION.-After premedication with atropine, general anaesthesia was induced. Intubation occasioned some difficulty, and there was anoxia for a short time coinciding with cardiac arrest. The baby was quickly brought into the operating theatre and the chest opened through the fifth left intercostal space. After cardiac massage the heart quickly started beating again. The other preliminaries to the operation were then completed. The incision was extended upwards across the third and second costal cartilages, then medially across the sternum and outwards into the second right intercostal space. This gave excellent exposure with a minimum of retraction.

The right ventricle was seen to be contracting well, in striking contrast to the left ventricle, which was pale and immobile and apparently replaced by fibrous tissue on the anterior and lateral aspects. The right coronary artery was immediately identified. It was of larger calibre than normal, and originated from the usual site on the aorta. It was very tortuous (Fig. 3), and several large branches were seen crossing towards the left side of the heart over the base of the right ventricle. Many large veins were also seen. The left coronary artery was small, and, after a difficult dissection, it was seen to emerge from the posterior aspect of the pulmonary artery about $1 \mathrm{~cm}$. above the posterior sinus. This dissection necessitated retraction of the pulmonary artery, which in turn caused interference with the pulmonary blood flow. As a result the heart beat was frequently weak, and at times completely arrested. On each occasion it was easily started again by relaxing the retraction on the pulmonary artery and squeezing the heart. On two occasions $1.5 \mathrm{ml}$. of $10 \%$ calcium chloride was given into the right ventricle. Fibrillation did not develop. Compression of the left coronary artery during dissection did not produce any ill effect on the heart; it was therefore totally occluded for five minutes while the electrocardiogram was carefully observed. No change in the heart beat or alteration in the elecrocardiogram occurred during this time, indicating that perfusion of this artery while anastomosing it with the subclavian would be unnecessary.

It had been hoped that the anomalous coronary artery would be found to originate from the more accessible left anterior sinus. Nevertheless, after further dissection of the back of the pulmonary artery.

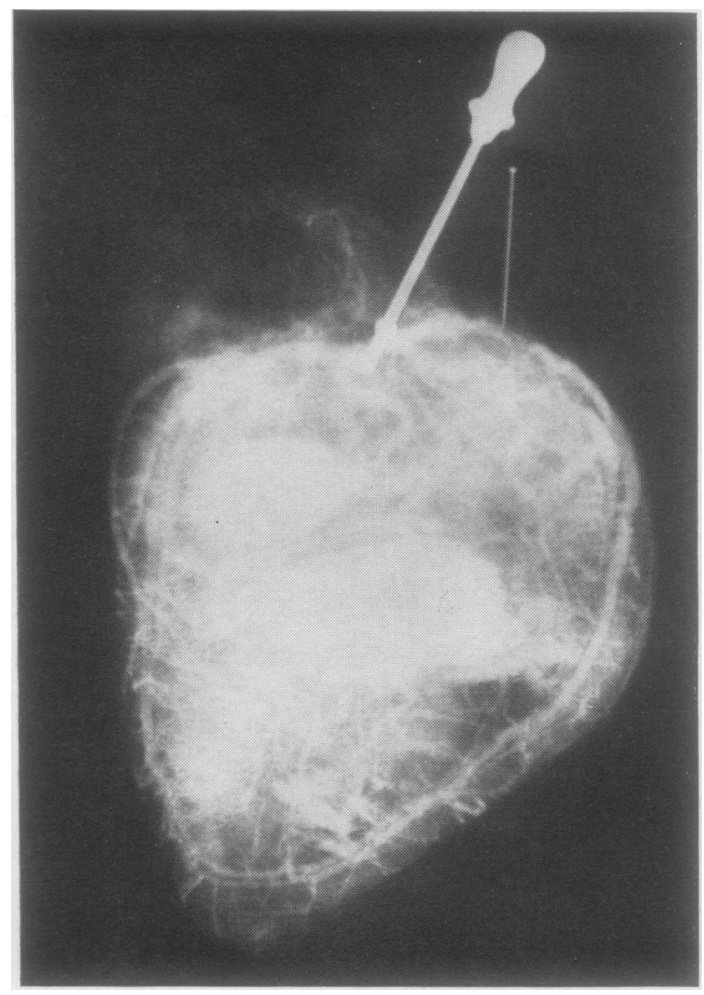

FIG. 4.-Radiograph of the heart after removal and injection of the right coronary artery with barium gelatine mixture. The anastomosis over the apex of the heart with the left coronary artery is well shown. 
it seemed possible to apply a clamp at the origin of the anomalous artery to include a disc of pulmonary artery large enough for anastomosis with the subclavian artery. Attention was then turned to the subclavian artery. It was dissected free to the point at which it left the chest, and proved long enough and large enough to be turned down to reach the coronary artery. Detachment of the coronary artery from the pulmonary artery was next attempted. Unfortunately it was not possible to apply the clamp satisfactorily, and a disc of pulmonary artery was not obtained all round the coronary artery, so that an anastomosis did not prove possible.

At this point it was noted that bright red blood was coming from the cardiac end of the left coronary artery. This suggested that it was, in fact, draining blood from the heart rather than carrying blood to the heart from the pulmonary artery. The coronary artery was therefore ligated, the pulmonary artery sutured, and the chest closed. Spontaneous respiration failed to be re-established, and the child died half an hour later.

\section{MorbiD ANATOMY}

When the heart had been removed from the body and was laid flat on its posterior surface, the empty left ventricle presented a concave appearance because the thin antero-lateral wall was collapsed into the lumen of the ventricle.

The heart was grossly enlarged and weighed $210 \mathrm{~g}$. The left ventricle was enormous $(9.5 \mathrm{~cm}$. long by $13.5 \mathrm{~cm}$. maximum circumference). A large part of the anterior and lateral walls of the left ventricle, including the apex, was replaced by scar tissue over an area of $8.5 \mathrm{~cm}$. by $8 \mathrm{~cm}$., which in the centre was transparent and $0.2 \mathrm{~cm}$. thick. The septum $(1.05 \mathrm{~cm}$.) and the posterior wall $(1.5 \mathrm{~cm}$.) of the left ventricle were thick and hypertrophied. The right coronary artery arose from the posterior aortic sinus and was a large vessel $0.3 \mathrm{~cm}$. in diameter. The left coronary artery arose from the posterior sinus of the pulmonary artery and was a thin-walled vessel about $0.15 \mathrm{~cm}$. in diameter. The right ventricle was somewhat hypertrophied. The remainder of the heart was normal.

The heart was injected through the right coronary artery, first with indian ink which stained the whole heart except the thin part of the left ventricle, and then with warmed barium gelatine mixture. Both these materials escaped during the injection from the origin of the left coronary artery. The heart was then rapidly cooled and radiographs were taken (Fig. 4). These showed vascular filling of the entire

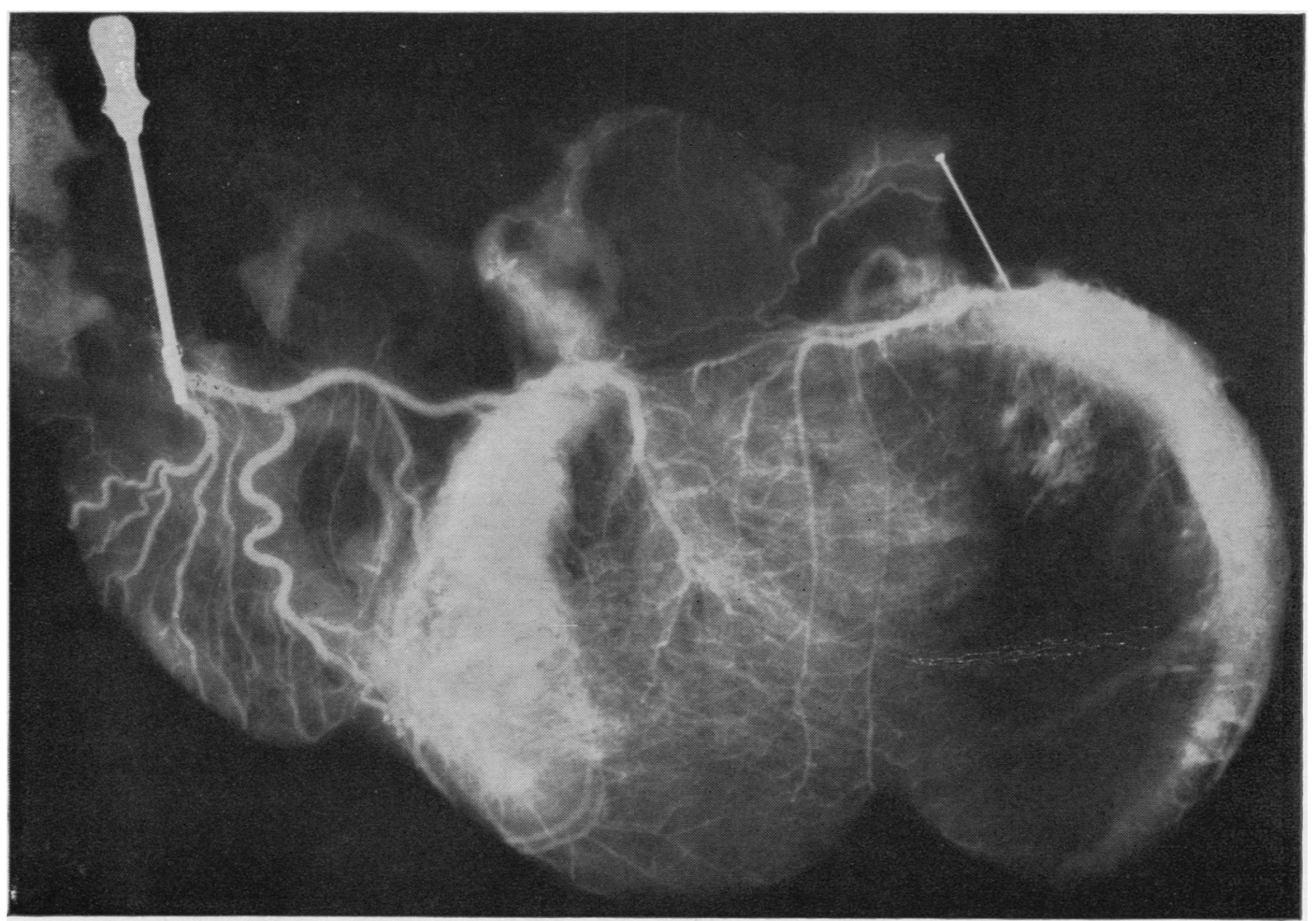

FIG. 5.-Radiograph of the heart after removal. The right coronary artery was injected with barium gelatine mixture; the cannula marks its origin. The origin of the left coronary artery is marked with a pin. The heart was cut and unrolled after Schlesinger's method. The avascularity of part of the left ventricle and some posterior communications between right and left coronary arteries are shown. 
heart wall except for the thin area of the left ventricle. Some veins were found to have filled with the mixture, and extravasation was also seen in the substance of the heart. The right and the anterior descending branches of the left coronary artery were seen to be continuous over the apex of the heart; large collaterals also connected the two vessels over the anterior aspect of the right ventricle at the base of the heart. There were also communications between the right and the circumflex branch of the left coronary arteries over the posterior aspect of the heart along the auriculo-ventricular groove.

The heart was then cut and unrolled according to Schlesinger's (1941) technique and again radiographed (Fig. 5). The extravasations were seen to be in the interventricular septum. The avascularity of the thinned part of the left ventricle was well shown. The anastomoses between the right and left coronary arteries, over the apex and the anterior aspect of the right ventricle, had been cut across, and their continuity was not demonstrated, but the other smaller communications are seen.

Histological examination of the anterior descending branch of the left coronary artery showed it to have a normal structure.

These studies therefore showed that all the heart muscle, with the exception of the thinned part of the left ventricle, was well supplied with blood from the right coronary artery and that communications between the right and left coronary arteries were extensive enough to allow considerable drainage of blood from the right coronary artery through the left coronary artery into the pulmonary artery. This possibility has previously been discussed by Edwards (1954). The large connexions between the two arteries, the absence of any electrocardiographic changes on clamping the left coronary artery at operation, and the fact that bright red blood flowed from the cardiac end when the artery was severed, lead us to consider that in this case the left coronary artery was acting as part of an arteriovenous fistula.

\section{Discussion}

No case of successful correction or alleviation of this anomaly has, as far as we are aware, yet been reported though several attempts have been made.

(1) The creation of an aortic-pulmonary fistula as a means of increasing the pressure and oxygen content of blood in the pulmonary artery and consequently in the left coronary artery was first suggested by Gasul and Loeffler in 1949. This has been attempted on two occasions by Potts (1955), but without success. It carries the disadvantage of increasing the load of work on the already damaged left ventricle.

(2) Elevation of pulmonary arterial pressure by constricting the pulmonary artery was suggested by Kittle and others (1955), who operated on an infant with this aim. The child died while the in- cision was being made and could not be resuscitated. The advantage of this method over the above is that no extra load is placed on the left ventricle. Kittle and others considered that in the coronary artery adequate pressure is more important than high oxygen saturation.

(3) Mustard (1953) made an attempt at direct $\overrightarrow{0}$ correction of the anomaly. He detached the por- $\overrightarrow{\vec{H}}$ tion of the pulmonary artery bearing the coronary $\vec{\omega}$ origin and anastomosed it to the left common carotid artery which he had turned down from the $\times$ neck. Perfusion of the coronary artery was main- $\vec{N}$ tained during the anastomosis through a polythene $\overrightarrow{-}$ tube with blood from a pump-oxygenator. The $\underset{\infty}{N}$ anastomosis was satisfactorily accomplished, but 은 the heart went into fibrillation at the end of the operation and could not be defibrillated. $\$ Hypothermia to $30^{\circ} \mathrm{C}$. was used during this operation, and may have contributed to the irreversible fibrillation.

This principle seemed to the authors to be the procedure of choice, and was adopted. It was intended to modify Mustard's actual technique by using the subclavian instead of the carotid, by tapping the aorta as a source for perfusion instead of using a pump-oxygenator, and by omitting the hypothermia. In retrospect it seems evident that the method used in this case was inappropriate. The cardiac disability was evidently due to the fact that a large part of the left ventricle was fibrous and not functioning, though the remainder of the left ventricular muscle was well supplied from the right coronary artery. The left coronary artery was found at operation to contain oxygenated blood, and it almost certainly drained blood from the right coronary artery into the pulmonary artery. Compression of the left coronary artery for five minutes did not produce any change in the electrocardiogram in this case. For these reasons it seemed that ligating it might produce some $\frac{7}{0}$ benefit. Clearly anastomosis of this artery to the systemic circulation would not have been helpful of in this case.

Gordon Murray (1947) carried out experiments on the excision of cardiac infarcts in dogs. $\mathrm{He}$ showed that systemic pressure and cardiac output are increased when the inert portion of ventricle $\stackrel{0}{\bar{D}}$ has been excised. He later (1950) successfully per- $\stackrel{\oplus}{\oplus}$ formed this procedure in a patient. Bailey (1955) has also successfully excised an aneurysm of the left ventricle in an adult following a coronary $\mathbb{D}$ occlusion. Resection of the infarct was probably $\frac{\Omega}{\mathbb{D}}$ feasible in the present case because there was $\cong$ sufficient remaining healthy muscle to form an adequate left ventricular chamber. 
From our experience in this case we consider it probable that excision of the infarct is the form of treatment most likely to be of benefit in this condition. It seems improbable that the anomaly can be diagnosed before the characteristic electrocardiographic changes have developed. By this time there is an established infarct and manipulation of the blood supply to the ventricle will not affect it ; the most that can be done in this respect is ligation of the anomalous artery to prevent drainage into the pulmonary artery. Surgery would not be appropriate where the collateral circulation to the left ventricle is adequate; such cases may survive into adult life (Brown, 1950), but a diagnosis is unlikely to be made in infancy.

\section{SUMMARY}

A case of anomalous left coronary artery is described in which an unsuccessful attempt was made to correct the anomaly.

Evidence is produced to show that the direction of blood flow in the anomalous artery was reversed.

It is suggested that, in view of the reversed blood flow demonstrated in the anomalous coronary artery and the irreversible local myocardial degeneration, correction of the anatomical defect is unlikely to produce improvement.
Reasons are given for concluding that excision of the cardiac infarct and ligature of the anomalous coronary artery is the appropriate treatment.

We are indebted to Dr. O. C. Lloyd, who performed the post-mortem examination; a part of the pathological description has been taken from his necropsy record.

We also wish to record our acknowledgments to Professor R. Milnes Walker for his helpful advice, to Dr. F. M. Ross for performing the radiological examination, and to Dr. R. Woolmer and Dr. J. Clutton Brock, who gave the anaesthetic.

\section{REFERENCES}

Abrikossoff, A. (1911). Virchows Arch. path. Anat., 203, 413. Bailey, C. P. (1955). Surgery of the Heart, p. 1043. Saunders, Philadelphia.

Barnard, W. G. (1938). J. Path. Bact., 47, 345. Bland, E. F., White, P. D., and Garland, J. (1933). Amer. Heart J.,

Brown, J. W. (1950). Congenital Heart Disease, 2nd ed., p. 96. Staples, London.

Edwards, J. E. (1954). Pediatric Clinics N. America (Feb.), p. 13.

Fisher, H., and Lloyd, O. C. (1951). Brit. Heart J., 13, 406.

Gasul, B. M., and Loeffler, E. (1949). Pediatrics, 4, 498.

Kittle, C. F., Diehl, A. M., and Heilbrunn, A. (1955). J. Pediat., 47, 198.

Murray, G. (1947). Ann. Surg., 126, 523.

(1950). In British Surgical Practice, vol. 8, p. 525. Butterworth, London.

Mustard, W. T. (1953). Personal communication.

Potts, W. J. (1955). Personal communication to Kittle et al., J. Pediat., 47, 198.

Schlesinger, M. J. (1941). J. Tech. Meth., $21,12$. 